\title{
CUANTIFICACIÓN INMUNOMAGNÉTICA DE CÉLULAS TUMORALES CIRCULANTES EN PACIENTES CON CÁNCER DE PRÓSTATA: CORRELACIÓN CLÍNICA Y PATOLÓGICA
}

\author{
L. Resel Folkersma, C. Olivier Gómez, L. San José Manso, S. Veganzones de Castro', \\ I. Galante Romo, M. Vidaurreta Lázaro', G.V.de la Orden', M. Arroyo Fernandez', E. Díaz \\ Rubio², A. Silmi Moyano y M. A. Maestro de las Casas.
}

Servicio de Urología. Servicio de Análisis Clínicos' y Servicio de Oncología². Hospital Universitario Clínico San Carlos. Madrid. España.

\begin{abstract}
Resumen.- OBJETIVOS: Detección y cuantificación de células tumorales prostáticas circulantes (CTC) en sangre periférica de pacientes con cáncer de próstata (CP) y estudiar la relación de las CTCS con los parámetros clínico-patológicos.
\end{abstract}

MÉTODOS: Estudio prospectivo con tres brazos: 26 pacientes ( $p$ ) con CP localizado (CPL); 24p con CP metastático (CPM) y 30 controles voluntarios sanos. Se extrajo una única muestra de $7,5 \mathrm{~mL}$ de sangre periférica y se aislaron las CTC según un método inmunomagnético basado en el sistema CellSearch (Veridex). Las CTCs fueron identificadas como células nucleadas negativas para el CD45 (leucocitos) y positivas para las citoque-

\section{CORRESPONDENCIA}

L. Resel Folkersman

Servicio de Urología

Hospital Universitario Clínico San Carlos

Martin Lagos, s/n

28040 Madrid. (España).

|rese|@gmail.com

Aceptado para publicar: 20 de octubre 2009 ratinas (8, 18 y 19). Se estudiaron las relaciones del número de CTCs con los niveles de PSA, Gleason y clasificación TNM.

RESULTADOS: Sólo el 10\% de controles sanos tenían 1 CTC/7,5 mL, ninguno de los pacientes con CP localizado tuvo más de 3 CTC $(88 \% \leq 2$ CTC) y aquellos con CPM presentaban niveles de CTCs significativamente más altos [m: 29 (1-178)] comparados con los otros dos grupos (P: 0.000). Se demostró una correlación positiva entre el número de CTC y cifras de PSA, con el tamaño del tumor y con la presencia o no de adenopatías. El grado Gleason fue el único parámetro que no mostró correlación con los niveles de CTC y aunque el número de CTC fue mayor en aquellos con metástasis viscerales [m: 297 (0-416)] comparado con los que tenían metástasis óseas [m: 68 (9,5-168)] estas diferencias no fueron significativas.

CONCLUSIONES: El análisis inmunomagnético nos permite cuantificar las CTC en sangre periférica y podría presentar una posibilidad para lograr una estadificación correcta y estimar un pronóstico adecuado de la enfermedad metastática.

Palabras clave: Células tumorales circulantes. Análisis inmunomagnético. Adenocarcinoma de próstata. Carcinoma metastático.

Summary.- OBJECTIVES: To detect and enumerate circulating prostatic tumor cells (CTC) in the peripheral blood of patients with prostate cancer (PC) and study the relationship between CTCs and clinical-pathological parameters. 
METHODS: Prospective three-arm study: 26 patients ( $p$ ) with localised PC (LPC); 24 P with metastatic PC (MPC) and 30 healthy volunteer controls. A single $7.5 \mathrm{ml}$ sample of peripheral blood was retrieved; CTCs were isolated using an immunomagnetic method based on the CellSearch system (Veridex). CTCs were identified as nucleated cells negative for CD45 (leukocytes) and positive for cytokeratins. (8, 18 y 19) The relationship between CTC numbers and PSA levels, Gleason score and TNM classification was studied.

RESULTS: Only 10\% of the healthy controls had 1 CTC $/ 7.5 \mathrm{~mL}$, none of the patients with localised PC had more than 3 CTCs $(88 \% \leq 2$ CTCs), and patients with MPC had significantly higher CTC levels [m: 29 1 1178)] compared with the other two groups (P: 0.000). A positive correlation was demonstrated between the CTC count and PSA levels, tumor size, and presen-ce or absence of enlarged lymph nodes. Gleason score was the only parameter that did not show any correlation with CTC levels, and although the number of CTCs was higher in patients with visceral metasta-ses [m: 29710 $4161]$ compared with bone metastases patients [m: 68 (9.5-168)], these differences were not significant.
CONCLUSIONS: Immunomagnetic analysis permits CTCs to be enumerated in peripheral blood and could be a possible way to correctly stage and make a reasonable prognosis of metastatic disease.

Keywords: Circulating. Immunomagnetic enrichment. Metastatic carcinoma. Prostatic carcinoma. Tumoral cells.

\section{INTRODUCCIÓN}

Las metástasis son la principal causa de morbilidad y mortalidad en pacientes con cáncer de próstata (CP). El concepto de metástasis engloba un complejo proceso de acontecimientos: las células neoplásicas del tumor primario atraviesan la membrana basal, penetran por las vías linfáticas y los vasos sanguíneos y se dispersan a tejidos distantes. La detección de células tumorales prostáticas circulantes (CTC) en sangre periférica puede tener importantes implicaciones pronosticas y terapéuticas (1).

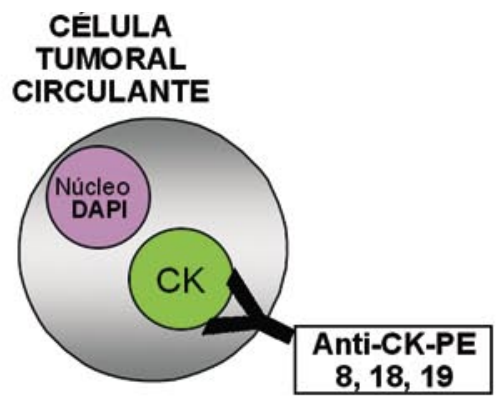

LEUCOCITO

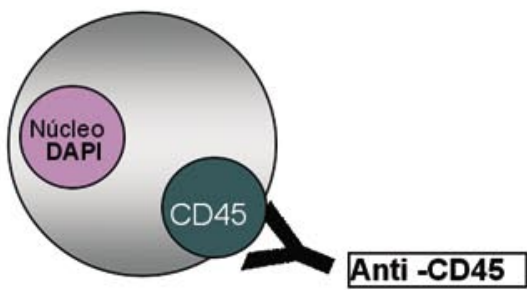

\section{CTC \\ CD45}
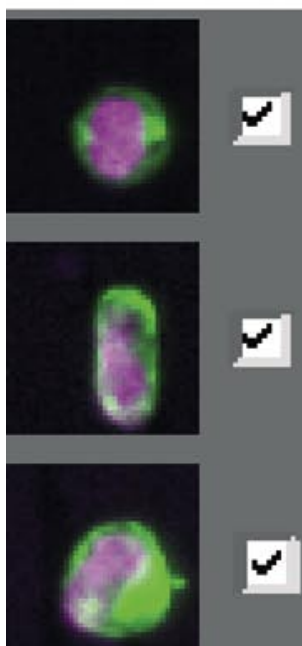
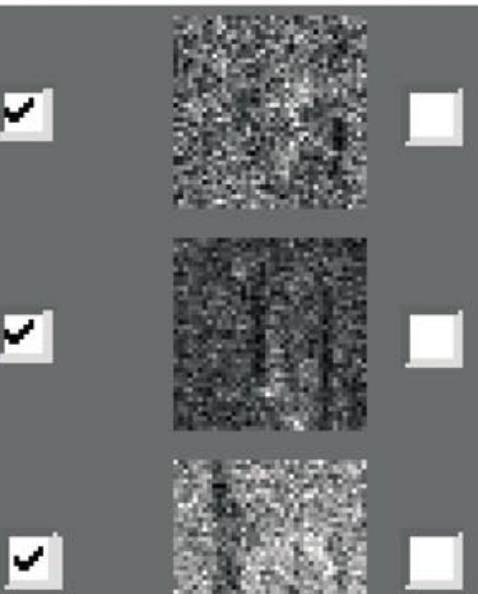

Control
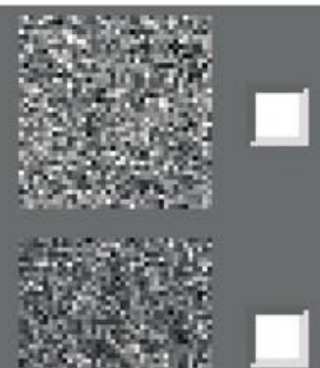
tos 504 arise 2005ing:
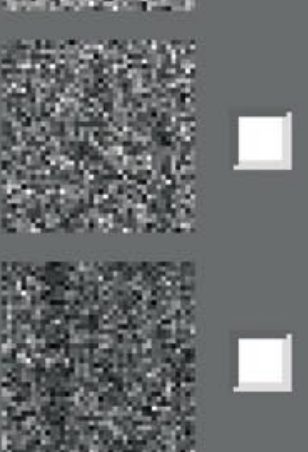

DAPI
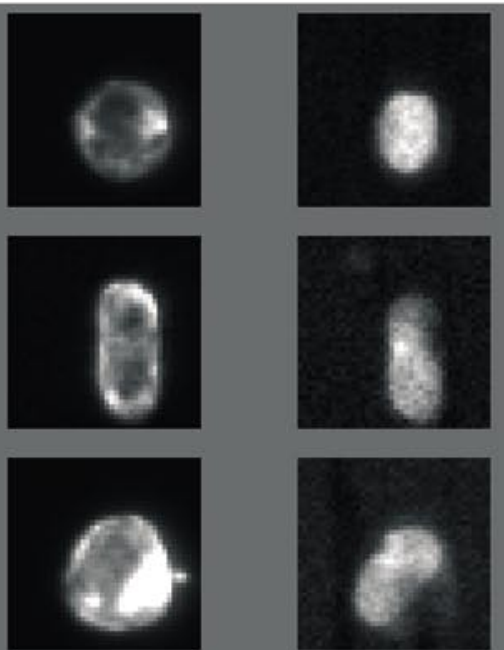

FIGURA 1. Inmunofenotipo de las células tumorales circulantes (DAPI+, CK-PEt, CD45-) y de los leucocitos (DAPIt,

CD45+, CK-PE-). En la imagen por debajo de las células muestra como se ven la CTCs con el microscopio de fluorescencia. 
Las experiencias preliminares en la determinación de CTC fueron atribuidas a Ashworh (2) quien, en 1869, describió el caso de un paciente diagnosticado de cáncer con células en sangre similares a las del tumor original. Aunque aparentemente su presencia podría implicar progresión de la neoplasia, su verdadero significado biológico no ha podido ser establecido debido a su escaso número y por la dificultad en el aislamiento e identificación con los procedimientos habituales. En los últimos años se ha producido un gran interés en la detección de células tumorales circulantes en tumores sólidos tanto en sangre periférica como en médula ósea. Esto es debido, en parte, al advenimiento de las técnicas de inmunohistoquímica, especialmente la reacción en cadena de la polimerasa de la transcriptasa inversa (RT-PCR). No obstante, los resultados de los diferentes estudios realizados con RT-PCR no han objetivado una clara asociación con el estadio patológico del CP (3), debido a que su valor esta limitado por la falta de estandarización de la técnica, por la posible contaminación de la muestra y fundamentalmente por su incapacidad para cuantificar las células tumorales.

Se ha desarrollado un nuevo sistema denominado CellSearch ${ }^{\text {TM }}$ de Veridex que nos permite la identificación y cuantificación de CTC en sangre periférica mediante análisis inmunomagnético (4) y que ha sido aceptado recientemente por la FDA para su determinación en pacientes con cáncer de mama, colon y próstata (5-7). Este método ya ha sido utilizado para aislar CTC en pacientes con cáncer de próstata metastático hormonorefractario en estudios previos y se ha demostrado que los niveles de CTC tienen un papel pronóstico en cuanto a la supervivencia de la enfermedad (7-10).

El objetivo principal de este estudio es la detección y cuantificación de los niveles de CTC en pacientes con CP localizado y metastático antes de iniciar un tratamiento, e investigar su relación con los niveles de PSA, grado Gleason y estadificación TNM.

\section{MATERIAL Y MÉTODO}

\section{Diseño del estudio}

El ensayo se diseñó como un estudio diagnóstico prospectivo realizado en nuestra institución entre mayo del 2006 y noviembre del 2008. El protocolo de investigación y el consentimiento informado fue aprobado por el Comité de ética de la institución y a todos los pacientes se les entregó un consentimiento informado por escrito. Se incluyeron tres grupos de
30 pacientes cada uno. Un grupo con CP localizado (CPL)(T1-T2, Clasificación TNM 2002), otro grupo con CP metastático (CPM)(T2-4 N1-2 Mla, b, c). El tercer grupo ha estado constituido por 30 voluntarios sanos menores de 40 años, sin antecedentes familiares de $\mathrm{CP}$ y con un antígeno prostático especifico (PSA) menor de $1 \mathrm{ng} / \mathrm{mL}$ en sangre periférica, asumiendo que la incidencia de $\mathrm{CP}$ en tales condiciones era muy baja.

El diagnóstico de CP se estableció mediante análisis histológico de la biopsia prostática en pacientes con CPM o de la pieza de prostatectomía radical en aquellos con CPL. Se establecieron como criterios de exclusión a todos aquellos pacientes diagnosticados de otro tumor o que estuvieran bajo tratamiento de supresión androgénica, además de cualquier paciente al que se le haya realizado una biopsia prostática 15 días antes de su inclusión en el estudio.

De acuerdo con las recomendaciones de la UICC todos los pacientes con CP fueron estudiados mediante tacto rectal, análisis de sangre general incluyendo el PSA (Tandem E, Hybritech Inc, San Diego, CA, USA) y ecografía transrectal (Siemens Sonoline G-50). Se realizó gammagrafía ósea y TAC abdominal o RNM para determinar la presencia de diseminación metastática.

\section{Aislamiento y cuantificación de CTC}

A cada paciente se le extrajeron dos muestras de $7,5 \mathrm{ml}$ de sangre periférica desde un mismo lugar de venopunción y después se introdujeron en tubos de preservación en vacío CellSave (Immunicon, Huntingdon Valley, Pa). Las muestras se mantuvieron a temperatura ambiente y fueron procesadas antes de las 72 horas de su recogida. Con la primera muestra se descartaban aquellas células epiteliales que se pudieran desprender por la propia venopunción y se utilizó para el análisis de sangre general. La segunda muestra se centrifugó a 2000 rpm durante 10 minutos y fue utilizada para la determinación de las CTC según el método CellSearch System (Veridex). Es un sistema semiautomático que usa el CellSearch Epithelial Cell Kit (Immunicon), se aíslan las células con fluido férrico y se marcan con anticuerpos dirigidos contra la molécula de adhesión que expresan la células epiteliales (anti-EpCAM). Las células epiteliales circulantes son aisladas mediante campos magnéticos y posteriormente teñidas con el acido nucleico fluorescente dye 4,2-diamidino-2phenylindole dihydrocloride (DAPI). Para distinguir las células epiteliales de los leucocitos se utilizaron anticuerpos monoclonales marcados con fluoresceína específicos para leucocitos (CD45-allophycocyan) 
y para células epiteliales [citoqueratinas 8, 18, 19 phycoerythrin (CK-PE)]. Las CTC fueron identificadas y enumeradas mediante el analizador CellSpotter, un microscopio semiautomático de fluorescencia que mediante un software específico permite reconocer a las células. Las CTC son definidas como aquellos objetos con apariencia de célula (ovalado con más de $3 \mathrm{~mm}$ de diámetro), nucleadas (DAPI+), con tinción positiva para citoqueratinas (CK-PE 8, 18, 19+) y que carecen de expresión para CD-45 (Figura 1). Los niveles de células tumorales circulantes se cuantificaron como número de CTC / 7,5 mL de sangre periférica.

\section{Análisis estadístico}

Se utilizó el test de la mediana para el estudio de los niveles de CTC y para estudiar su relación con las adenopatías regionales (N), asumiendo un nivel de significación estadística de 0,05. Las variables se resumen en medianas y rangos intercuartílicos $\left(\mathrm{P}_{25}-\mathrm{P}_{75}\right)$.

Para valorar las relaciones entre los niveles de CTC y las cifras de PSA, Gleason y estadificación TNM del CP se utilizaron los coeficientes de corre-

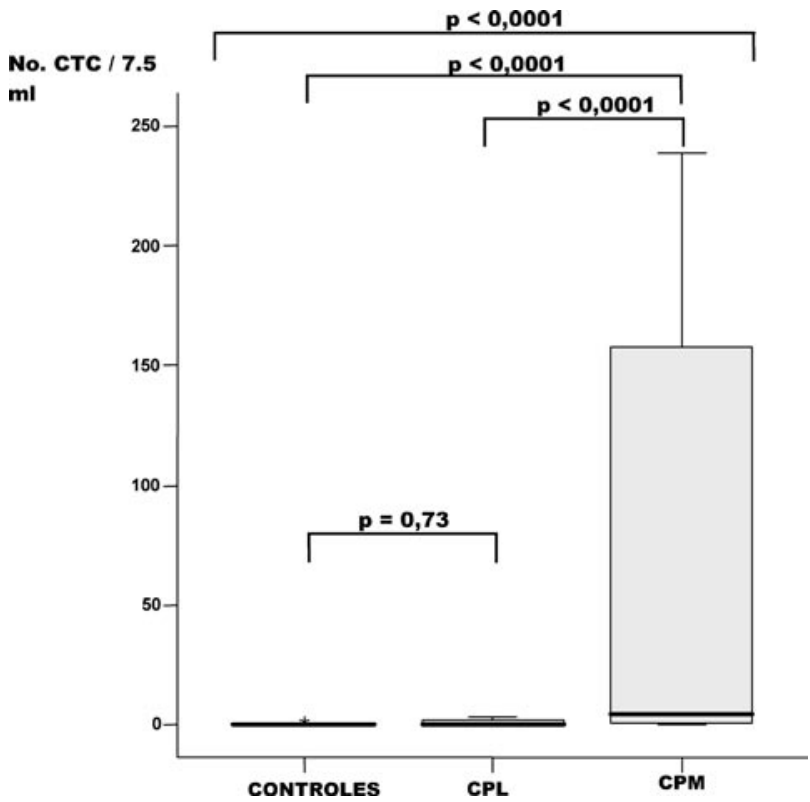

FIGURA 2. Comparación de los niveles de CTC entre los diferentes grupos estudiados. Se observaron diferencias estadísticamente significativas entre los 3 grupos ( $p<0,0001)$, sin embargo no se encontraron diferencias significativas entre los controles sanos y el grupo con adenocarcinoma prostático localizado (p:

$$
\text { 0,73). }
$$

lación Tau de Kendall y Rho de Spearman según el caso.

En todos los contrastes de hipótesis se rechazó la hipótesis nula con un error de tipo I o error $\alpha$ menor a 0,05.

El análisis estadístico se realizó con el programa SPSS 15.0.

\section{RESULTADOS}

Un total de 80 pacientes fueron reclutados en nuestra institución entre mayo del 2006 y noviembre del 2008. Cuatro pacientes pertenecientes al grupo de adenocarcinoma de próstata localizado (CPL) fueron retirados debido a un error en el procesamiento de la sangre (muestra insuficiente, coagulada) y 6 pacientes del grupo de CP metastático (CPM) se rechazaron del estudio porque fueron diagnosticados de otro tumor durante el estudio. Los datos demográficos y niveles de PSA se muestran en la Tabla I, en la tabla II se reflejan los datos clinicopatológicos de aquellos pacientes diagnosticados de CPL y CPM.

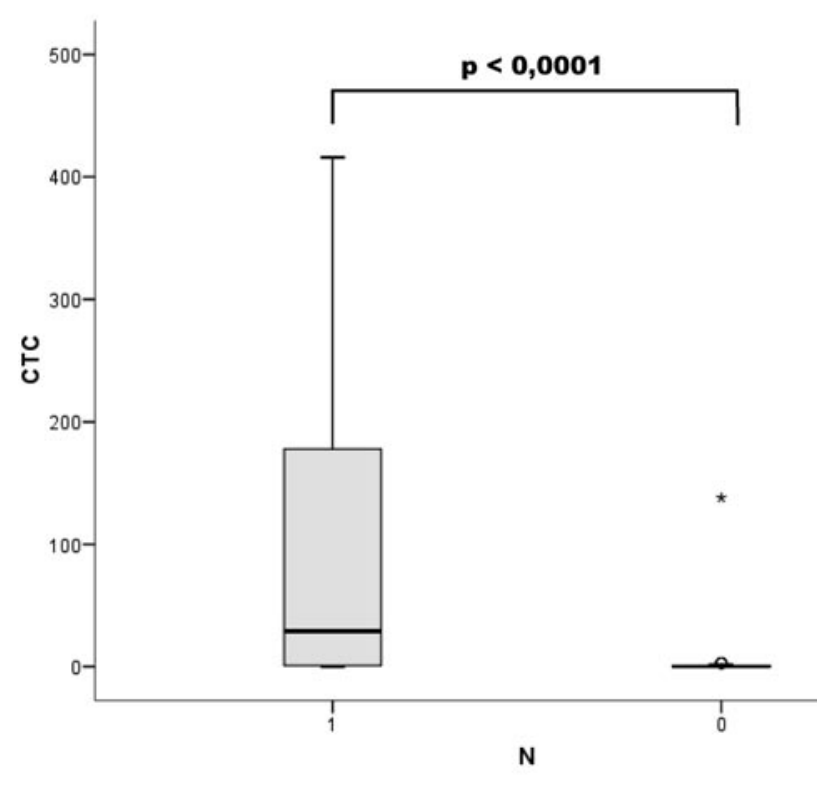

FIGURA 3. Comparación de los niveles de CTC entre los pacientes con (N1) o sin adenopatías regionales (NO). Los niveles de CTC fueron significativamente más altos en pacientes con adenopatías regionales [mediana: 29 (50-226)] ( $P<0,0001)$, comparado con aquellos pacientes en los que no se evidenció la presencia de adenopatías [mediana: 0,0 (0,0-1)] $(\mathrm{P}<$ 0,00011 . 


\section{TABLA I. DATOS DEMOGRÁFICOS Y NIVELES DE PSA DE LOS DIFERENTES GRUPOS.}

\begin{tabular}{|c|c|c|c|}
\hline & CONTROL & CPL & CPM \\
\hline Número & 30 & 26 & 24 \\
\hline Edad & $32(29-38)$ & $64(54-77)$ & $73(53-91)$ \\
\hline PSA $(\mathrm{ng} / \mathrm{mL})$ & $0.49(0.2-1.1)$ & $4.87(2.16-14.6)$ & $485(41.73-2435)$ \\
\hline
\end{tabular}

El tratamiento efectuado a todos los pacientes con CPL consistió en prostatectomía radical abierta, laparoscópica o robótica. A los pacientes con CPM se les instauró tratamiento mediante bloqueo hormonal después de incluirles en el estudio. Al tiempo de realizar el estudio, tres de los pacientes pertenecientes al grupo de CPM fallecieron (12,5\%) (2 de los pacientes murieron por progresión de la enfermedad y uno por sepsis a la semana del diagnóstico).
En el grupo CPL, tres pacientes diagnosticados inicialmente como $\mathrm{T} \leq 2$ fueron posteriormente $\mathrm{T} 3$ [2T3a y $1 \mathrm{~T} 3 \mathrm{~b}$ ] (se obtuvo un 10\% de infraestadiaje) al analizar la pieza de prostatectomía, uno de estos pacientes con CPL T3a tuvo el rango superior de CTC en este grupo ( $3 \mathrm{CTC}$ ), en los dos restantes no se obtuvieron CTC en sangre periférica.

En 7 pacientes clínicamente diagnosticados de CP y con evidencia de MTS óseas (grupo CPM) no

TABLA II. GRADO GLEASON Y ESTADIFICACIÓN SEGÚN LA CLASIFICACIÓN TNM 2002.

\begin{tabular}{|c|c|c|c|}
\hline & & CPL (\%) & CPM (\%) \\
\hline \multirow[t]{3}{*}{ GLEASON } & $<=7$ & $25(96)$ & $7(41.1)$ \\
\hline & 8 & 0 & $4(23.5)$ \\
\hline & $9-10$ & $1(4)$ & 5 (29.4) \\
\hline \multirow{6}{*}{$\mathrm{T}$} & $\mathrm{T} 2 \mathrm{a}$ & 11 (42.3) & 0 \\
\hline & $\mathrm{T} 2 \mathrm{~b}$ & $3(11.5)$ & 0 \\
\hline & $\mathrm{T} 2 \mathrm{c}$ & $8(30.7)$ & $1(4.1)$ \\
\hline & $\mathrm{T} 3 \mathrm{a}$ & $3(11.5)$ & $6(25)$ \\
\hline & $\mathrm{T} 3 \mathrm{~b}$ & $1(4.5)$ & 9 (37.5) \\
\hline & $\mathrm{T} 4$ & 0 & $3(12.5)$ \\
\hline \multirow{3}{*}{$N$} & $\mathrm{Nx}$ & 0 & 0 \\
\hline & NO & $26(100)$ & $2(8.3)$ \\
\hline & N1 & 0 & $22(91.6)$ \\
\hline \multirow{3}{*}{$M$} & Mla & 0 & $3(12.5)$ \\
\hline & $M 1 b$ & 0 & $18(75)$ \\
\hline & Mlc & 0 & $3(12.5)$ \\
\hline
\end{tabular}


se pudo obtener la histología por complicaciones en su estado general.

Los niveles de CTC lexpresados en n CTC / $7,5 \mathrm{ml}$ ) se resumen en la Tabla III.

En el grupo de controles voluntarios sanos, sólo en el 10\% se encontró una CTC / 7,5 mL y en ninguno de los pacientes diagnosticados de CP localizado se obtuvieron más de 3 CTC $(11,5 \%, 3 / 26)$, de hecho el $88,4 \%$ tuvo $\leq 2$ CTC. En el grupo de CPM la mediana fue de 29 CTC / 7,5 mL, hubo un $71 \%(17 / 24)$ que tuvieron un número $\geq$ de 2 CTC, si establecíamos el punto de corte en $>=5$ CTC el porcentaje disminuyó a $58 \%$ y si lo establecíamos en $\geq$ de 3 CTC apenas variaba y se quedaba en un $66,6 \%(16 / 24)$.

Las diferencias en cuanto al número de CTC entre los tres grupos evaluados, controles sanos, CPL y $C P M$, fueron estadísticamente significativas $\left(P_{<}\right.$ $0,0001)$. Sin embargo no hubo diferencias de los niveles de CTC entre los voluntarios sanos y el grupo con CPL (P: 0,738). Los niveles de CTC fueron significativamente más altos en pacientes con $\mathrm{CP}$ metastásico [mediana: 29 (1-178)] comparado con aquellos pacientes con CP localizado [mediana: $0,0(0,0-1)$ ] $(P<0,0001)$ y controles sanos [mediana: $0,0(0,0-$ $0,0)](P<0,0001)$ (Figura 2).

Cuando se comparaban los niveles de CTC con los diferentes parámetros clínico-patológicos, se observaron diferencias significativas en el número de CTC entre los que presentaban o no adenopatías regionales $(N)(P=0,01)$ (Figura 3), se demostró una buena correlación positiva entre los niveles de CTC y cifras de PSA (Rho=0,59, $p<0,0001)$ y las metásta- sis (Tau=0,56, $p<0,0001$ ) respectivamente (Figura 4). Aunque el número de CTC era progresivamente mayor en los subgrupos Mla (adenopatías no regionales), Mlb (lesiones óseas) y Mlc (afectación visceral) no se evidenciaron diferencias significativas $(p=0,319)$ (Figura 5). También se demostró una modesta correlación positiva entre el número de CTC y el tamaño del tumor (T)(Tau=0,45, $p<0,0001$ ) (Figura 4), el grado de Gleason fue el único parámetro con el que no se encontró correlación (Tau=0,23, $\mathrm{p}=0,07$ ).

\section{DISCUSIÓN}

La presencia de células metastáticas ocultas en pacientes diagnosticados de tumor es de una gran importancia, ya que la diseminación precoz de células tumorales es una de las principales causas de recidiva y de mortalidad por cáncer (11-13). Estudios previos han sugerido que la presencia de CTC en pacientes con metástasis originadas por neoplasias de cólon (6, $14)$, mama $(5,15)$ y cáncer de próstata hormonoresistente $(8,10,16,17)$, se asocia a una supervivencia libre de progresión y global más corta.

Entre las técnicas que se han empleado para el aislamiento y caracterización de las CTC, figura la RTPCR (18), pero ha obtenido resultados contradictorios. Así, en algunos estudios se ha conseguido correlacionar de forma significativa los niveles de RT-PCR para el PSA en la sangre periférica tanto con el estadiaje clínico como patológico del CP (19-23), pero en otros centros no se han podido corroborar dichos datos (3, 24, 25). Esta disparidad de resultados puede estar relacionada con la complejidad en el procesamiento de la muestra, que requiere múltiples pasos y con la dificultad para cuantificar los resultados.

TABLA III. NIVELES DE CTC EN LOS TRES GRUPOS.

\begin{tabular}{|c|c|c|c|}
\hline CTC $/ 7,5 \mathrm{~mL}$ & CONTROL & CPL & CPM \\
\hline Mínimo & 0 & 0 & 0 \\
\hline Máximo & 1 & 3 & 919 \\
\hline p25 & 0.0 & 0.0 & 1.0 \\
\hline p50 & 0.0 & 0.0 & 29 \\
\hline p75 & 0.25 & 1.0 & 178 \\
\hline
\end{tabular}

Niveles de CTC en los tres grupos expresados en valores absolutos (número mínimo y máximo de CTC), medianas (p50) y rangos intercuartílicos (p25-p75). 

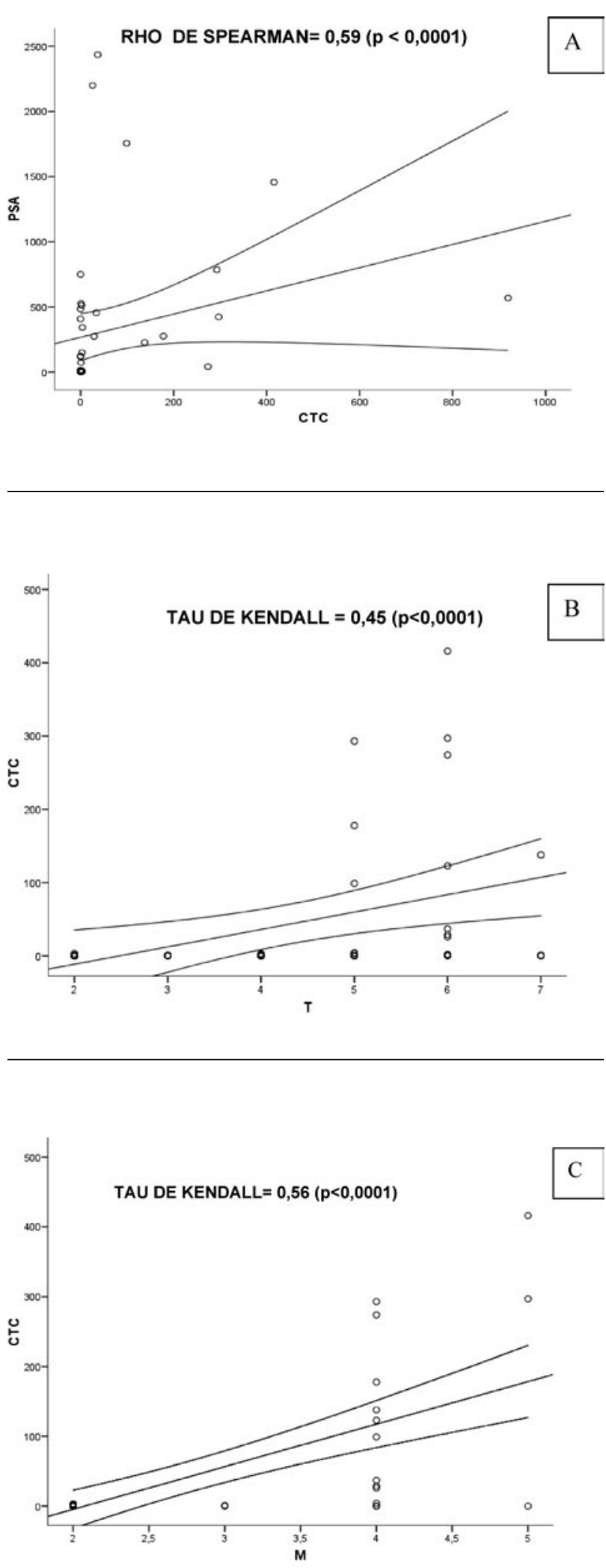

FIGURA 4. Estudio de la correlación entre los niveles de CTC con los marcadores de carga tumoral. A: Asociación de CTC con PSA (Rho de Spearman=0,59, p<0,000 1); B: CTC con el tamaño del tumor (T) I Tau de Kendall=0,45, p<0,0001); C: CTC con las metástasis (Tau de Kendall=0,56, $p<0,0001$ ).
Gracias al método inmunomagnético, la capacidad para cuantificar y caracterizar las CTC en pacientes con CP es ya una realidad clínica (5-10), este sistema es semiautomático, apto para realizar en un laboratorio clínico y por lo tanto perfectamente reproducible. El sistema de análisis CellSearch fue diseñado para detectar células epiteliales en sangre periférica. Se ha publicado que las anormalidades cromosómicas obtenidas en las CTC aisladas de pacientes con neoplasias epiteliales con MTS equivalen con las encontradas en las células tumorales de la lesión primaria (26), lo que indica que dichas células circulantes derivaban del tumor. En otro reciente estudio sobre CP hormonorresistente, Shaffer et al. (9) han demostrado que dichas CTC son verdaderamente neoplásicas y que pueden ser estudiadas para determinar tanto su nivel de proteínas como los cambios cromosómicos mediante técnicas de fluorescencia con hibridación in situ.

El número de CTC para considerar que una muestra es positiva en pacientes con $\mathrm{CP}$ metastático queda todavía por determinar. En un estudio prospectivo multicéntrico doble ciego, Cristofanilli et al. (5) establecieron un valor de corte de $5 \mathrm{CTCs} / 7,5$ $\mathrm{mL}$ en pacientes con cáncer de mama metastático, para demostrar que los niveles de dichas CTCs antes de iniciar la terapia, e incluso más importante, después en el seguimiento, eran útiles predictores de la supervivencia libre de progresión y de la supervivencia global. Moreno et al. (7) instauraron como valor de corte en pacientes con CP metastático el tener 2 o más CTC, además demostraron que el número de CTC no variaba a lo largo del día. Chen et al. (8) utilizaron el mismo valor de corte en pacientes con CP avanzado ya que al utilizar el valor de corte de 5 CTC la tasa de pacientes con CTC disminuyó de $62 \%$ al $42 \%$. En otros estudios más recientes en los que se ha considerado como resultado positivo el aislamiento de 5 o más CTC los porcentajes oscilaban entre el $57-65 \%$ (9-10). Sólo existe un estudio en el que se haya estudiado el número de CTC en pacientes con CP localizado, realizado por Moreno et al. (7), en el que obtuvieron un número muy variable de CTC (116, media de 5,9 CTC), los principales inconvenientes son que el número de pacientes era muy reducido (10 pacientes), el rango de PSA variaba de 0,2 a $22,6 \mathrm{ng} / \mathrm{mL}$ y no había una confirmación anatomopatológica del estadiaje.

En el presente estudio no hubo ningún voluntario sano que tuviera más de una CTC al igual que Moreno et al. (7), en aquellos pacientes con CP localizado tampoco hubo ninguno que sobrepasara el nivel de más de 3 CTC y cuando aplicábamos este nivel de corte para los pacientes con CPM, el porcentaje disminuía ligeramente al $66 \%$ (71\% con más de 


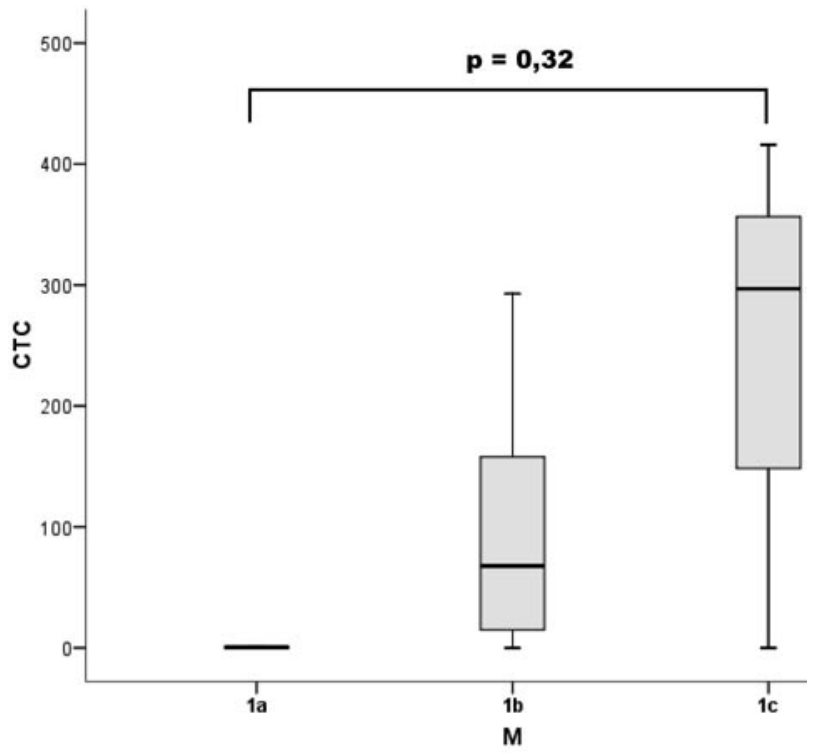

FIGURA 5. Comparación de los niveles de CTC entre los pacientes con metástasis (M). Los niveles de CTC fueron más altos en los pacientes con metástasis viscerales (M Ic) [mediana: 297 (0-4 16)], comparado con aquellos pacientes con metástasis óseas (M lb) [mediana: $68(9,5-168)$ ] y con adenopatias no regionales (M la) [mediana: 0,5 (0-7)], sin embargo dichas diferencias no fueron significativas $(p=0,32)$.

2 CTC). El seguimiento posterior de nuestros pacientes podría esclarecer este punto.

De todas formas, es evidente que, dada la escasez de estudios a este respecto para poder determinar el valor de corte adecuado, son necesarios más análisis y estudios prospectivos con un mayor número de pacientes.

Aunque el nivel de CTC está considerado como un biomarcador pronóstico importante para la supervivencia de la enfermedad metastática, su relación con los diferentes parámetros que nos informan de la extensión de la enfermedad no tiene una significación clínica clara. Chen et al. (8) demostraron una correlación positiva entre los niveles de CTC y los niveles de PSA, fosfatasa alcalina y bajo nivel de hemoglobina. Sin embargo el grado Gleason (p $=0.351)$ y la presencia de metástasis óseas o viscerales $(p=0.085)$ no predijeron la presencia de CTCs. Shaffer et al. (9) demostraron que a pesar de que existía una fuerte asociación entre el nivel de CTC y la supervivencia, cuando estudiaban su relación con el PSA y el número de metástasis óseas (bone scan index), la relación fue baja. En nuestro estudio encontramos una asociación significativa entre los niveles de CTC y el PSA, tamaño del tumor y la presencia de adenopatías y metástasis, siendo la correlación más fuerte la que se estableció con el PSA $(R h o=0,59)$ y las metástasis (Thau=0,56). No se encontró una relación significativa entre el grado Gleason y el número de CTC, y a pesar de la diferencias encontradas entre el número de CTC y la localización de las metástasis óseas o viscerales (el nivel de CTC era más alto en las MTS viscerales), éstas no fueron significativas. Quizás con un mayor número de pacientes estas diferencias llegarían a ser significativas, pero esto, al igual que los otros parámetros previos analizados, todavía quedan por esclarecer en estudios posteriores.

Aunque los resultados son prometedores, el aislamiento y cuantificación de células tumorales en sangre periférica no aborda el potencial biológico de dichas CTCs. La diferente distribución en cuanto al nivel de CTC en pacientes con CP metastático que podía variar de 0 a 919 CTC / 7,5 mL $(29 \%$ de pacientes con CPM tenían $\leq 2$ CTC) a pesar de la evidencia de enfermedad diseminada parece confirmar la heterogeneidad en el comportamiento biológico de la neoplasia prostática. Las diferentes vías de diseminación de la enfermedad tumoral metastática podrían estar en relación con estos hallazgos, además, la entrada en el torrente circulatorio es solo un paso en el proceso de diseminación metastática y puede no ser el evento más importante para producir metástasis en el CP.

\section{CONCLUSIONES}

El sistema de análisis inmunomagnético es un buen método para la detección y cuantificación de células tumorales prostáticas circulantes en sangre periférica.

Se ha demostrado una asociación positiva de los niveles de CTC con todos los marcadores de carga tumorales estudiados excepto con el grado Gleason, por lo que la determinación de CTC en pacientes con CP podría representar una posibilidad para poder lograr una estratificación correcta, estimar el pronóstico, instaurar terapias precoces y evaluar la respuesta a los diferentes tratamientos realizados.

\section{BIBLIOGRAFÍA Y LECTURAS RECOMENDADAS ("lectura de interés $y^{* *}$ lectura fundamental)}

*1. Ghossein RA, Bhattacharya S and Rosai J. Molecular detection of micrometastases and circulating tumor cells in solid tumors. Cin Cancer Res 1999; 5:1950-60. 
2. Ashworth TR. A case of cancer in wich cells similar to those in the tumours were seen in the blood after death. Australian Med J 1869; 14:146.

**3. Llanes L, Ferruelo A, Lujan M, Pascual C, Garcia-Mediero JM and Berenguer A. Quantitative real-time reverse transcription: polymerase chain reaction of prostate-specific antigen (PSA) for detection of circulating prostatic cells in patients with clinically localized prostate cancer. Prostate Cancer and Prostatic diseases 2005; 8:248-52.

4. Kagan M, Howard D, Bendele T, Rao C and Terstappen LW. A sample preparation and analysis system for identification of circulating tumor ce1ls. J Clin Ligand Assay 2002; 25:104-10.

**5. Cristofanilli M, Budd T, Ellis MJ, Stopeck A, Matera J, Miller MC et al. Circulating tumor cells, disease progresión, and survival en metastático breat cancer. N Engl J Med 2004; 351:781-91.

6. Hardingham JE, Hewett PJ, Sage RE, Finch JL, Nuttall JD, Kotasek D et al. Molecular detection of blood-bone epithelial cells in colorectal cancer patients and in patients with benign bowel disease. Int J Cancer 2000; 89:8-13.

*7. Moreno JG, O’Hara SM, Gross S et al. Changes in circulating carcinoma cells in patients with metastatic prostate cancer correlate with disease status. Urology 2001; 58:386-92.

**8. Chen BT, Loberg RD, Neeley CK, O’Hara SM, Gross S, Doyle G et al. Preliminary study of immunomagnetic quantification of circulating tumor cells in patients with advanced disease. Urology 2005; 65(3):616-21.

**9. Shaffer DR, Leversha MA, Danila DC, Lin O, Gonzalez-Espinoza R, Gu B et al. Circulating tumor cell analysis in patients with progresive castration-resistant prostate cancer. Clin Cancer Res 2007; 13(7):2023-29.

**10. Danila DC, Heller G, Gignac GA, Gonzalez-Espinoza R, Anand A, Tanaka E et al. Circulating tumor cell number and prognosis in progressive castration-resistant prostate cancer. Clin Cancer Res 2007; 13(23):7053-57.

*11. Braun S, Pantel K, Müller P, Janni W, Hepp F, Kentenich CR et al. Cytokeratin-positive cells in the bone marrow and survival of patients with stage I, II or III breast cancer. N Engl J Med 2000; 342:525-33.

12. Hellman S. Stopping metastases at their source. $\mathrm{N}$ Engl J Med 1997; 337:996-7.

13. Black RJ, Bray F, Ferlay J and Parkin DM. Cancer incidence and mortality in the European Union: cancer registry data and estimates of national incidence for 1990. Eur J Cancer 1997; 33:1075-107.

*14. Sastre J, Maestro ML, Puente J, Veganzones S, Alfonso R, Rafael $\mathrm{S}$ et al. Circulating tumor cells in colorrectal cancer: correlation with clinical and pathological variability. Ann Oncol 2008; 19:935-38.
**15. Budd GT, Cristofanilli M, Ellis MJ, Stopeck A, Borden E, Miller MC et al. Circulating tumor cells versus imaging-predicting overall survival in metastatic breast cancer. Clin Cancer Res 2006; 12:6403-09.

**16. Moreno JG, Miller MC, Gross S, Allard WJ, Gomella LG and Terstappen LW. Circulating tumor cells predict survival in patients with metastatic prostate cancer. Urology 2005; 65:713-18.

*17. García JA, Rosenberg JE, Weinberg V, Scott J, Frohlich M, Park JW et al. Evaluation and significance of circulating epithelial cells in patients with hormone-refractory prostate cancer. BJU Int 2007; 99:519-24.

18. Makarovskiy AN, Ackerley W 3rd, Wojcik L, Halpert GK, Stein BS, Carreiro MP et al. Application of immunomagnetic beads in combination with RT-PCR for the detection of circulating prostate cancer cells. J Clin Lab Anal 1997; 11:346-50.

19. Katz AE, Olsson CA, Raffo AJ, Cama C, Perlman $\mathrm{H}$, Seaman E et al. Molecular staging of prostate cancer with the use of an enhanced reverse transcriptase-PCR assay. Urology 1994; 43:765-75.

20. Okegawa T, Noda H, Kato M, Miyata A, Nutahara $\mathrm{K}$ and Higashihara $\mathrm{E}$. Value of reverse transcription polymerase chain reaction assay in pathological stage T3N0 prostate cancer. Prostate 2000; 44:210-8.

*21. Zhang Y, Zippe CD, Van Lente F, Klein EA and Gupta MK. Combined nested reverse transcription-PCR assay for prostate-specific antigen and prostate-specific membrane antigen in detecting circulating prostatic cells. Cin Cancer Res 1997; 3:1215-20.

*22. Israeli RS, Miller WH Jr, Su SL, Samadi DS, Powell CT, Heston WD et al. Sensitive detection of prostatic hematogenous micrometastases using PSA and PSM-derived primers in the polymerase chain reaction. J Urol 1995; 153:573-7.

23. Grasso YZ, Gupta MK, Levin HS, Zippe CD and Klein EA: Combined nested RT-PCR assay for PSA and PSMA in prostate cancer patients: correlation with pathological stage. Cancer Res 1998; 58:1456-9.

24. Gomella LG, Raj GV and Moreno JG. Reverse transcriptase polymerase chain reaction for prostate specific antigen in the Management of prostate cancer. J Urol 1997; 58:326-37.

25. De la Taille A, Olsson CA and Katzt AE. Dream or reality?. Oncology 1999; 13:187-205.

**26. Fehm T, Sagalowsky A, Clifford E, Beitsch P, Saboorian $\mathrm{H}$, Euhus D et al. Cytogenetic evidence that circulating epithelial cells in patients with carcinoma are malignant. Clin Cancer Res 2002; 8:2073-84. 